\title{
Editorial
}

Digestion

\section{Autoimmune Pancreatitis: Avoiding Unnecessary Procedures by Following International Consensus Guidelines}

\author{
Georg Beyer $^{\mathrm{a}}$ Volker Ellenrieder $^{\mathrm{b}} \quad$ Albrecht Neesse $^{\mathrm{b}}$

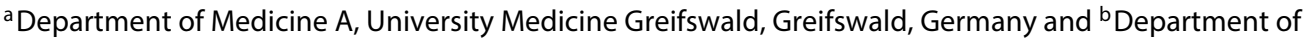 \\ Gastroenterology and Gastrointestinal Oncology, University Medical Centre Göttingen, Göttingen, Germany
}

The diagnostic and therapeutic management of autoimmune pancreatitis (AIP) has recently received increasing scientific and clinical attention in the gastroenterology community. AIP is a rare pancreatic disorder that poses great diagnostic challenges since clinical presentation can hardly be distinguished from other more frequent forms of pancreatitis or pancreatic cancer. By international consensus, we now distinguish 2 clinical subtypes of AIP, which despite the common name, in fact are 2 distinctly different entities [1-3]. AIP type 1 predominantly occurs in Asia, is mostly found in elderly men and can be diagnosed without the use of histologic sampling in about $70 \%$ of the cases in experienced centers [4]. It is the pancreatic manifestation of a systemic autoimmune disorder termed 'IgG4-related disease', with multiorgan involvement (kidney, salivary glands, biliary tree, retroperitoneum, lymph node and prostate) and the hallmark feature of IgG4-positive plasma cell infiltrates and elevated IgG4 plasma levels in the majority of patients. The histological presentation is described as lymphoplasmic sclerosing pancreatitis, and painless obstructive jaundice is the most common clinical symptom. In contrast, AIP type 2 is a disease that is limited to the pancreas and usually presents with characteristic painful pancreatitis episodes. AIP type 2 is often diagnosed in younger Western European patients and can be associated with inflamma- tory bowel disease. Importantly, IgG4 serum levels are typically not elevated and other organ involvement does not occur in AIP type 2 [5]. Therefore, histologic proof of 'idiopathic duct centric pancreatitis' with granulocyte epithelial lesions is required to definitely make the diagnosis of AIP type 2 [2].

In 2011, the International Consensus Diagnostic Criteria (ICDC) for AIP was published as an attempt to incorporate the various existing diagnostic guidelines from around the world. Briefly, the ICDC employs 5 key features for the diagnosis of AIP: pancreatic imaging, serology, other organ involvement, histology and steroid responsiveness that are graded in level 1 and 2 evidence.

In this issue of Digestion, Dr. Manser et al. [6] carefully analyzed the diagnostic workup in 29 Swiss patients with AIP, which had been prospectively collected since 2006. Using ICDC, the authors show that more than onethird of endoscopic retrograde cholangiopancreaticographies (ERCPs) and endoscopic ultrasounds (EUSs) could have been avoided if the diagnostic algorithm suggested by the ICDC had been followed. EUS or ERCP was deemed unnecessary if the diagnosis of AIP could have been established by imaging, histology or IgG4 serum levels, or if a cancer workup had not been indicated/resulted negative. Furthermore, 8 patients were referred for unnecessary pancreatic surgery from other hospitals.

\section{KARGER 125}

(c) 2015 S. Karger AG, Base

$0012-2823 / 15 / 0923-0171 \$ 39.50 / 0$
Albrecht Neesse, MD, PhD

Department of Gastroenterology and Gastrointestinal Oncology University Medical Centre, Georg August University Göttingen Robert-Koch-Strasse 40, DE-37099 Göttingen (Germany)

E-Mail albrecht.neesse@med.uni-goettingen.de 
Despite the relatively small study population, this paper is very interesting for the gastroenterology community as it addresses several issues that clinicians may encounter during clinical decision making.

First, jaundice was the single risk factor associated with unnecessary procedures. Although endoscopic or surgical interventions are often indicated in patients with obstructive jaundice, it is mostly not required in AIP patients. The fact that the latest estimations have shown that the incidence of AIP is about 10 times lower than that of pancreatic cancer makes this a particularly challenging call [7]. Therefore, rigorous use of the ICDC will help to reduce misdiagnosis and endoscopic or surgical 'over'treatment of AIP. However, if ERCP is still performed due to diagnostic uncertainty, the endoscopist should be aware of the 4 pancreatographic features that - isolated or combined - are highly suggestive for AIP, namely (i) long ( $>1 / 3$ the length of the pancreatic duct) strictures with a (ii) lack of upstream dilatation $(<5 \mathrm{~mm})$ or (iii) multiple strictures [8]. Unfortunately, the Swiss data collection did not analyze these features in detail.

From an epidemiological point of view, the study by Manser et al. [6] reveals an unusually high percentage of AIP type 1 that one would not expect in a Western European patient population. However, histological confirmation was not provided in most of the AIP cases which might have affected the ultimate classification of AIP type 1 or type 2 .

Notably, typical imaging findings (sausage-like, diffusely swollen pancreas) that have been described for the majority of Asian patients with AIP type 1 were only detected in $20 \%$ of the Swiss cohort. Although the study population was preselected due to a high percentage of referred patients from smaller hospitals, the clinical and radiographic demonstration of AIP in Western Europe might be more heterogeneous and complex than previously anticipated and may depend on multiple factors, for example, time duration after onset of initial symptoms. Therefore, histological evidence provided by EUSfine needle aspiration (EUS-FNA) provides the most accurate diagnosis and should be strongly advocated if AIP cannot be diagnosed by the non-invasive ICDC criteria.

To conclude, the study by Manser et al. [6] shows several interesting and clinically relevant aspects for the diagnostic management of AIP patients. First, the ICDC offer a comprehensive strategy for the workup of patients with suspected AIP. Rigorous employment of ICDC may help to avoid or at least reduce the number of unnecessary procedures and surgery. Second, typical imaging findings of AIP might be less frequent than initially described in the literature, thus further complicating the correct diagnosis of AIP. In particular, focal AIP may be an obstacle for finding the correct diagnosis by imaging modalities and EUS-FNA [9]. As a consequence, further refinements and consequent use of EUS-FNA with subsequent assessment by reference pathologists should be strongly endorsed in cases where suspected AIP cannot be diagnosed by non-invasive tests.

\section{Disclosure Statement}

No conflict of interest declared.

\section{References}

1 Chari ST, Kloeppel G, Zhang L, Notohara K, Lerch MM, Shimosegawa T: Histopathologic and clinical subtypes of autoimmune pancreatitis: the Honolulu consensus document. Pancreatology 2010;10:664-672.

2 Shimosegawa T, Chari ST, Frulloni L, Kamisawa T, Kawa S, Mino-Kenudson M, et al: International consensus diagnostic criteria for autoimmune pancreatitis: guidelines of the international association of pancreatology. Pancreas 2011;40:352-358.

3 Hart PA, Zen Y, Chari ST: Recent advances in autoimmune pancreatitis. Gastroenterology 2015;149:39-51.
4 Sah RP, Chari ST: Autoimmune pancreatitis: an update on classification, diagnosis, natural history and management. Curr Gastroenterol Rep 2012;14:95-105.

5 Kamisawa T, Chari ST, Giday SA, Kim MH, Chung JB, Lee KT, et al: Clinical profile of autoimmune pancreatitis and its histological subtypes: an international multicenter survey. Pancreas 2011;40:809-814.

6 Manser $\mathrm{CN}$, et al: Unnecessary procedures and surgery in autoimmune pancreatitis. Digestion 2015;92:138-146.

7 Umehara H, Okazaki K, Masaki Y, Kawano M, Yamamoto M, Saeki T, et al: A novel clin- ical entity, IgG4-related disease (IgG4RD): general concept and details. Mod Rheumatol 2012;22:1-14.

8 Sugumar A, Levy MJ, Kamisawa T, Webster GJ, Kim MH, et al: Endoscopic retrograde pancreatography criteria to diagnose autoimmune pancreatitis: an international multicentre study. Gut 2011;60:666-670.

9 Mauch M, Blank W, Kunze G, Dirks K, Schuler A, Klinggräff von $\mathrm{G}$, et al: Importance of abdominal ultrasound in 17 patients with histologically confirmed autoimmune pancreatitis (AIP). Ultraschall Med 2015;36:248254 